\title{
Astragalus membranaceus/Angelica gigas/Trichosanthes kirilowii Maximowicz Mixed Herbal Extract SHOO3
}

National Cancer Institute

\section{Source}

Nationa/ Cancer Institute. Astragalus membranaceus/Angelica gigas/Trichosanthes

kirilowii Maximowicz Mixed Herbal Extract SH003. NCl Thesaurus. Code C148489.

A traditional Chinese medicine (TCM)-based herbal extract composed of Astragalus membranaceus (Am), Angelica gigas (Ag) and Trichosanthes kirilowii Maximowicz (Tk), with potential anti-angiogenic and antineoplastic activities. Upon administration, the active ing redients in $\mathrm{Am} / \mathrm{Ag} / \mathrm{Tk}$ mixed herbal extract SH003 work synerg istically to exert a number of activities through various mechanisms of action (MOA); although, not all of the MOAs are fully elucidated. SH003 blocks the binding of vascular endothelial growth factor (VEGF) to its receptor VEGF receptor 2 (VEGFR2; KDR), thereby inhibiting VEGF/VEGFR2-mediated signaling and VEGF-induced tumor endothelial cell mig ration, invasion and tube formation. This inhibits tumor angiogenesis. In addition, SHOO3 inhibits signal transducer and activator of transcription 3 (ST AT3) activation and ST AT 3mediated signaling, decreases the production of the pro-inflammatory cytokine interleukin-6 (IL-6), and the expression of STAT3 target genes. This induces apoptosis in, and reduces proliferation and metastasis of, cancer cells in which STAT 3 signaling is overactivated. ST AT 3 activation contributes to inflammation in the cancer environment and tumor cell proliferation. 